Prediction of functi onal recovery after revascul ar i zat i on usi ng quant i t at i ve gat ed myocar di al perfusi on SPECT: a mil ti - cent er cohort study i n Japan

\begin{tabular}{|l|l|}
\hline 著者 & $\begin{array}{l}\text { Nakaj i ma Keni chi, Nagar a Tamaki, Kuwabar a } \\
\text { Yoi chi, Kawano Nasaya, Nat sunar i I chi ro, Taki } \\
\text { Juni chi, N shi mur a Shi geyuki, Yamashi na Aki ra, } \\
\text { I shi da Yoshi o, Tombi ke H t onobu }\end{array}$ \\
\hline $\begin{array}{l}\text { j our nal or } \\
\text { publ i cat i on t i t l e }\end{array}$ & $\begin{array}{l}\text { Eur opean Jour nal of Nucl ear Nedi ci ne and } \\
\text { Nol ecul ar I magi ng }\end{array}$ \\
\hline vol une & 35 \\
\hline nunber & 11 \\
\hline page r ange & 2038 - 2048 \\
\hline year & 2008 11- 01 \\
\hline URL & ht t p: //hdl . handl e. net /2297/11733 \\
\hline
\end{tabular}




\title{
Prediction of functional recovery after revascularization using quantitative gated myocardial perfusion SPECT: a multi-center cohort study in Japan
}

\author{
Kenichi Nakajima ${ }^{1}$, Nagara Tamaki ${ }^{2}$, Yoichi Kuwabara ${ }^{3}$, Masaya Kawano ${ }^{4}$, Ichiro Matsunari ${ }^{5}$, Junichi Taki ${ }^{6}$, \\ Shigeyuki Nishimura ${ }^{7}$, Akira Yamashina ${ }^{8}$, Yoshio Ishida ${ }^{9}$ and Hitonobu Tomoike ${ }^{9}$ \\ 1. Department of Biotracer Medicine, Kanazawa University Graduate School of Medical Science, Kanazawa, Japan \\ 2. Department of Nuclear Medicine, Hokkaido University Graduate School of Medicine, Sapporo, Japan \\ 3. Department of Cardiovascular Science and Medicine, Chiba University Graduate School of Medicine, Chiba, Japan \\ 4. Departments of Radiology, Kanazawa Cardiovascular Hospital, Kanazawa, Japan \\ 5. The Medical and Pharmacological Research Center Foundation, Hakui, Japan \\ 6. Department of Nuclear Medicine, Kanazawa University Hospital, Kanazawa, Japan \\ 7. Department of Cardiology, Saitama Medical University, Saitama, Japan \\ 8. Department of Cardiology, Tokyo Medical University, Tokyo, Japan \\ 9. National Cardiovascular Center, Osaka, Japan
}

For Kanazawa University Repository, Accepted for publication in Eur J Nucl Med Mol Imaging 2008 DOI 10.1007/s00259-008-0838-6

Corresponding Author:

Kenichi Nakajima, MD, Department of Biotracer Medicine, Kanazawa University Graduate School of Medical Science, 13-1 Takara-machi, Kanazawa, 920-8641, Japan, Phone: +81-76-265-2333, Fax: +81-76-234-4257,

Email: nakajima@med.kanazawa-u.ac.jp

\section{Abstract}

Backgrounds Prediction of left ventricular functional recovery is important after myocardial infarction. The impact of quantitative perfusion and motion analyses with gated single-photon emission computed tomography (SPECT) on predictive ability has not been clearly defined in multi-center studies.

Methods A total of 252 patients with recent myocardial infarction $(n=74)$ and old myocardial infarction $(n=175)$ were registered from 25 institutions. All patients underwent resting gated SPECT using ${ }^{99 \mathrm{~m}}$ Tc-MIBI, and repeated the study after revascularization after an average follow-up period of $132 \pm 81$ days. Visual and quantitative assessment of perfusion and wall motion were performed in 5040 segments.

Results Non-gated segmental \%uptake and end-systolic (ES) \%uptake were good predictors of wall motion recovery and significantly differed between improved and non-improved groups $(66 \pm 17 \%$ and $55 \pm 18 \%, \mathrm{p}<0.0001$ for non-gated, $64 \pm 16 \%$ and $51 \pm 17 \%$ for ES \%uptake, $\mathrm{p}<0.0001$ ). The area under the curve (AUC) of receiver-operating characteristics curve (ROC) for non-gated \%uptake, ES \%uptake, end-diastolic (ED) \%uptake and visual perfusion defect score was $0.70,0.71,0.61$ and 0.56 , respectively. Sensitivity and specificity of \%uptake were $68 \%$ and $64 \%$ for non-gated map and $80 \%$ and 52\% for ES \%uptake map. An optimal threshold for predicting segmental improvement was $63 \%$ for non-gated and $52 \%$ for ES \%uptake values.

Conclusion Segmental ${ }^{99 \mathrm{~m}}$ Tc-MIBI uptake provided a useful predictor of wall motion improvement. Application of quantitative approach with non-gated and ES \%uptake enhanced predictive accuracy over visual analysis particularly in a multi-center study.

Keywords: ${ }^{99 m}$ Tc-hexakis-2-methoxy-isobutyl isonitrile (MIBI), viability, gated single-photon emission computed tomography, multi-center cohort study, coronary revascularization

\section{Introduction}

Predicting functional recovery before revascularization in patients with known coronary artery disease is important to determine indications for percutaneous coronary intervention (PCI) and coronary artery bypass grafting (CABG). Numerous experimental and clinical studies have demonstrated that when episodes of ischemia results in regional contractile dysfunction, restoration of perfusion will result in improvement in regional contractility. Even if some myocardial segments showed severe contractile dysfunction, left ventricular global and regional contractility may recover owing to viable segments. Non-invasive myocardial viability assessment thus has been considered important, and various approaches have been utilized including contractile functional reserve by echocardiography with inotropic stimulation, myocardial perfusion imaging with single-photon emission computed tomography (SPECT) and positron emission tomography with ${ }^{18} \mathrm{~F}$-fuluorodeoxyglucose (FDG) [1-6]. Although FDG may provide better diagnostic ability for detecting potentially reversible myocardial dysfunction, single-photon myocardial perfusion tracers continue to be one of the best convenient choices for clinical practice. Prediction of regional functional recovery was successfully achieved 
by ${ }^{99 \mathrm{~m}}$ Tc-hexakis-2-methoxy-isobutyl isonitrile (MIBI) SPECT as well as by ${ }^{201} \mathrm{Tl}$, and viable and non-viable segments could be differentiated [7-12].

Considering that many nuclear medicine facilities can perform gated SPECT, a quantitative approach for gated SPECT analysis has become a common diagnostic procedure [13]. Thus, prediction of viability using all available parameters would be potentially beneficial by the current use of quantitative gated SPECT. Currently available techniques for predicting functional recovery after revascularization was summarized by Bax et al. in 1997, and 37 studies were summarized [5]. The numbers of patients in each of the ten MIBI studies were less than 40, and only a few studies dealt with $>100$ patients even after that point on [12]. In addition, the studies were performed in a single center, and few multicenter studies have been performed. Thus, we designed a multi-center cohort study with participation of Japanese hospitals for predicting recovery of ventricular dysfunction using resting ${ }^{99 \mathrm{~m}}$ Tc-MIBI SPECT. The goal of this study was to determine the best predictor and threshold for viability assessment with quantitative analysis as well as visual segmental analysis, and apply them as a practical clinical indicator for revascularization.

\section{Materials and Methods}

Patients

This study group for viability assessment began in July 2003 and final follow-up SPECT ended in March 2006. This research was a multi-center cohort study, prospectively performed. A total of 262 patients were registered from 25 institutions throughout Japan. The principal criteria for patient selection consisted of myocardial infarction documented by coronary angiography associated with left ventricular wall motion abnormality, and the patients were scheduled for coronary intervention. The inclusion criteria were (1) recent myocardial infarction (RMI) 7 to 30 days from the onset of myocardial infarction or old myocardial infarction (OMI, defined as $>30$ days after onset) and (2) existence of regional wall motion abnormality before revascularization, (3) successful reperfusion without restenosis until the second post-intervention SPECT study, and (4) informed consent received from the patients. A pre-intervention SPECT study and coronary angiography should have been performed within one month of revascularization, and post-intervention follow-up SPECT was performed $<1$ year after the revascularization. However, during the follow-up and summary periods, 10 patients were excluded from the study, which included those with no segments of significant asynergy found in subsequent analysis $(n=3)$, and segments of asynergy found only from the non-revascularized artery segments $(n=5)$. In addition, because of a technical error during data transfer, data from 2 patients could not be analyzed. Finally, 252 patients (216 males and 36 females) were judged optimal for subsequent data analysis (Table 1). The mean age was $67.1 \pm 11.1$ years. Out of the 252 patients,
74 (29\%) were diagnosed with RMI, 175 (69\%) with OMI, and $3(1 \%)$ with both RMI and OMI. The study protocol was approved by either the Review Board or the Ethical Committee of the participating institutes. Written informed consent was obtained from all patients who participated.

\section{Coronary arteriography and intervention}

Coronary arteriography was performed in each hospital with standard techniques, and the coronary segments and stenosis were judged based on American Heart Association (AHA) criteria. The stenosis of coronary arteriography $\geq 75 \%$ was judged significant. The number of vessels involved was 140 one-vessel, 59 two-vessel and 53 three-vessel diseases (Table 1). Percutaneous coronary intervention (PCI) was performed in 210 patients $(83 \%)$ and coronary artery bypass grafting (CABG) in 40 patients $(16 \%)$. Localization of infarction was right coronary artery (RCA) in 117 patients (46\%), left anterior descending artery (LAD) in $150(60 \%)$ and left circumflex artery (LCX) in $70(28 \%)$. The presence of restenosis was judged by the combination of clinical information that included coronary arteriography $(\mathrm{n}=178,71 \%)$, stress and rest myocardial perfusion SPECT, exercise and resting electrocardiography (ECG) and clinical signs and symptoms.

\section{Gated SPECT study}

An ECG-gated perfusion SPECT was obtained at rest using ${ }^{99 \mathrm{~m}}$ Tc-MIBI with a standard administration dose (300-1110 MBq). Gated SPECT was performed in all patients before and after revascularization. All institutes used a standard data acquisition protocol for the MIBI study, but the precise acquisition conditions were based on their routine settings. Out of 25 institutes, 20 used a two-detector system, and 5 used a three-detector system. A low-energy high-resolution collimator was used in 11 institutes, a low-energy general-purpose collimator in 10 institutes and a low-medium-energy general-purpose collimator in 4 institutes. The camera rotation range was 360 degrees $(n=14)$ and 180 degrees $(n=11)$. All institutes used $64 \times 64$-matrix projection images. Pixel size ranged from 5.2 to $6.9 \mathrm{~mm}$. The number of division per cardiac cycle was $16,12,10$ and 8 frames in $12,4,1$ and 8 institutes, respectively. Rejection of arrhythmia was used in 17 institutes. Attenuation correction was performed in 2 institutes. A combination of Butterworth and ramp filters was used in all hospitals.

\section{SPECT data processing and image interpretation}

All projection images were transferred with a DICOM (Digital Imaging and Communications in Medicine) format to the core laboratory. Since multiple vendors were involved in the SPECT acquisition and reconstruction, the projection images were again reconstructed using the same reconstruction parameters (eNTEGRA workstation, GE Yokogawa Medical Systems, Ltd., Tokyo). The cutoff frequency of the Butterworth filter was 0.4 cycles/cm for gated images 
and $0.4-0.5$ cycles $/ \mathrm{cm}$ for non-gated images with a slight difference depending on the image counting statistics. After reconstruction of the gated short-axis slices, QGS software and QPS software (Cedars Sinai Medical Center, CA, USA) were used for visual interpretation and subsequently for quantitative analysis. Myocardial segments were divided into 20 segments with QGS and QPS, namely six basal segments, six mid segments, six apical segments in the short-axis slices, and 2 apical segments in the mid vertical long-axis slice [14]. The 20-segment model was validated well and found to show good correlation with a 17-segment model [15]. Correspondence of coronary artery territory to segments was RCA to the apical inferior, mid and basal inferoseptal and inferior segments (5 segments); LCX to the apical, mid and basal anterolateral and inferolateral segments (6 segments); and LAD to the remaining segments covering anterior, septal and the apex (9 segments). Regarding perfusion defects, defect scores were visually analyzed with five-point scoring in each segment; namely, normal (0), slightly decreased (1), moderately decreased (2), severely decreased (3), and complete defect (4). Regional wall motion was similarly analyzed with five-point scoring in 20 segments; namely, normokinesis (0), mild hypokinesis (1), severe hypokinesis (2), akinesis (3) and dyskinesis (4). The wall motion was interpreted using only motion display of the short-axis and vertical long-axis views without information of quantitative analysis.

\section{Interobserver variability of interpretation}

The existence of perfusion defect and segmental asynergy was visually judged in the core laboratory of Kanazawa University by four nuclear medicine specialists. A total of 5040 segments were analyzed by these four specialists. Quantitative data from QGS and QPS were hidden for the visual image interpretation. Before interpretation, four interpreters gathered several times, interpreted the same sets of sample studies, and discussed how to reach the uniform five-grade scoring criteria. In addition, 760 segments of 38 studies were analyzed independently by four interpreters, and inter-observer reproducibility was examined. Based on this test run, interobserver agreement among the four interpreters (6 combinations) showed weighted kappa values of 0.81 ( $95 \%$ confidence interval $[\mathrm{CI}], 0.78-0.83)$ to $0.86(0.84-0.88)$ for perfusion defect scores and $0.75(0.72-0.78)$ to 0.82 (0.80-0.84) for wall motion scores.

\section{Quantitative analysis}

All of the quantitative analyses were performed using the same software and conditions in one laboratory. Quantitative perfusion data used average perfusion values (\%) in 20 segments by QPS software. Wall motion ( $\mathrm{mm}$ ) and wall thickening (\%) were also calculated by QGS software. A normal database pre-installed in QPS and QGS was not used, and only average values in each segment were utilized for the segmental analysis.
Analysis for viability assessment

Regarding parameters for predicting motion improvement, non-gated \%uptake values on QPS and end-systolic (ES) and end-diastolic (ED) \%uptake values on QGS were used. Visual scoring was performed without these quantitative data. The abnormal wall motion before intervention was defined by a visual motion score of 2 to 4 and/or a quantitative thickening value of $\leq 32 \%[16,17]$. We used wall thickening values in the 20 -segment model as an adjunct for asynergy assessment. After intervention, since CABG was included in $17 \%$ of cases, septal motion showed abnormality even with good wall thickening [18, 19]. We therefore determined that the motion was improved after reperfusion if the percentage of wall thickening was improved to $>32 \%$. The segments having abnormal wall motion in the reperfused coronary territory were analyzed. Number of viable segments defined by the appropriate threshold count, which was $63 \%$ for non-gated map and 52\% for ES map based on the results, was examined as a patient-based predictor for $E F$ improvement.

\section{Statistical analysis}

The results were expressed by mean and standard deviation (SD). The inter-observer reproducibility of the regional wall motion score and visual perfusion score were assessed using a weighted kappa value and their 95\% confidence intervals, with quadratic weighting assignment [20]. Statistics of sensitivity, specificity, accuracy, and positive and negative predictive values for predicting wall motion improvement were calculated with respect to the perfusion parameters. Receiver operating characteristics curves (ROCs) were generated, and the area under the curve (AUC) and optimal threshold were determined. The ROC curve was also analyzed to determine AUCs in subgroups of coronary artery stenosis and revascularization. The significance of the differences in cardiac function between before and after revascularization was assessed by paired t test. The significance of the differences in viability markers between improved and non-improved groups was assessed by unpaired $t$ test for parametric variables and Wilcoxon rank-sum test for non-parametric variables. To compare AUCs among different predictors, a nonparametric method was used [21]. Multiple comparison tests were adjusted with a Bonferroni correction. All statistical tests were two-tailed, and a $\mathrm{p}$ value of $<0.05$ was considered significant. SAS System Release 8.2 for Windows was used for statistical analyses.

\section{Results}

Improvement of regional segmental asynergy

At least one mild hypokinesis was observed in 125 patients (50\%), severe hypokinesis in 187 patients (74\%), akinesis in 113 patients (45\%) and dyskinesis in 20 patients (8\%). A total of 1730 segments $(33 \%)$, in which coronary revascularization was 
performed, were analyzed. Segmental asynergy was improved in 360 segments $(21 \%)$ and not improved in 1370 segments $(79 \%)$. The \%uptake values on non-gated, ES and ED perfusion maps are summarized in Table 2. Before revascularization, non-gated \%uptake was $66 \pm 17 \%$ and $55 \pm 18 \%$ for improved and non-improved groups, respectively $(\mathrm{p}<0.0001)$. The ES \%uptake was $64 \pm 16 \%$ and $51 \pm 17 \%$ for improved and non-improved groups, respectively $(\mathrm{p}<0.0001)$. When \%uptake was compared before and after revascularization in the improved group, it improved by $+9 \%,+10 \%$ and $+3 \%$ units for non-gated, ES and ED perfusion maps, respectively $(\mathrm{p}<0.0001)$. In contrast, the \%uptake improved by $+3 \%$ units for non-improved groups using the three maps. The \%wall thickening increased by $+14 \%(\mathrm{p}<0.0001)$ in the improved group, while it increased by only $+1 \%$ in the non-improved group. Average perfusion defect score decreased from 1.0 to 0.3 in the improved group $(p<0.0001$ by Wilcoxon test). In the non-improved group, the mean score slightly decreased from 1.3 to 0.9 after revascularization $\quad(p<0.0001)$. Before the revascularization, visual perfusion defect score was slightly smaller in the improved group than in the non-improved group $(\mathrm{p}=0.0006)$.

Figure 1 shows a patient with old myocardial infarction in the anteroseptal and inferior walls, and perfusion and thickening were improved after coronary stenting. He had risk factors of hypertension, diabetes mellitus and current smoking. EF increased from $35 \%$ to $53 \%$, and EDV was improved from 163 to $135 \mathrm{~mL}$.

ROC analysis for the predictors of motion improvement

When diagnostic ability was evaluated by the parameters of \%uptake by non-gated perfusion and ES and ED perfusion maps, the AUCs were 0.70, 0.71 and 0.61 , respectively, whereas the AUC by visual scoring was the lowest (0.56) (Figure 2). The AUC of both non-gated and ES \%uptake was significantly higher than that of ED \%uptake $(p<0.0001)$. No significant difference was found between non-gated and ED \%uptake maps.

When the AUC was calculated in each coronary artery territory regarding \%uptake on non-gated perfusion map, LAD, LCX and RCA territories were $0.71,0.66$ and 0.73 , respectively and that on the ES perfusion map was $0.71,0.70$ and 0.73 , respectively (Table 3). The RMI patients showed slightly lower AUC value than OMI patients. Patients who underwent revascularization in 1, 2 and 3 vessels showed similar AUC values of 0.71-0.72 for non-gated perfusion map, and $0.74,0.72$ and 0.70 for ES perfusion map. The type of revascularization, namely PCI and CABG, showed AUC values from 0.70 to 0.73 and did not differ significantly.

Diagnostic accuracy based on the optimal threshold

The optimal threshold value was determined by the point on the ROC curve where the sum of sensitivity and specificity was maximal. When the optimal \%uptake of $63 \%$ was used for the non-gated perfusion map, the sensitivity, specificity and accuracy for predicting wall motion recovery were $68 \%, 64 \%$ and $65 \%$, respectively (Table 4 ). When an optimal \%uptake of $52 \%$ was used for the ES perfusion map, they were $80 \%, 52 \%$ and $58 \%$, respectively. The AUC was comparable for the non-gated map (0.70) and \%ES map (0.71). When optimal \%uptake of $43 \%$ on the ED perfusion map was used, they were $63 \%, 54 \%$ and $56 \%$, respectively. For visual analysis with the optimal cutoff of score 1 , the sensitivity, specificity and accuracy were $70 \%, 41 \%$ and $47 \%$.

\section{Changes of global function after revascularization}

Ejection fraction (EF) in this study ranged from $11 \%$ to $81 \%$, with an average of $50 \% \pm 15 \%$. Average EFs were significantly improved after revascularization: $50 \pm 15 \%$ and $53 \pm 14 \%$ before and after revascularization, respectively ( $\mathrm{p}<0.0001$, paired $\mathrm{T}$ test). Average EDV and ESV were $103 \pm 47 \mathrm{~mL}$ and $56 \pm 42$ $\mathrm{mL}$ in the first study, and the volumes were significantly reduced to $96 \pm 45 \mathrm{~mL}$ and $49 \pm 37 \mathrm{~mL}$ after revascularization, respectively ( $p<0.0001$ for both).

When non-gated \%uptake and ES \%uptake were compared with respect to baseline EF before revascularization and improvement in $\mathrm{EF}>5 \%, 28 \%$ of patients with baseline $\mathrm{EF} \geq 50 \%$ and $42 \%$ of patients with EF $<50 \%$ showed improvement (Table 5). EF and wall thickening were also written for comparison. In the group of improved EF, mean EF and mean \%thickening increased significantly $(p<0.0001)$. When the segment of minimal \%uptake was observed in this group, both mean non-gated and ES \%uptake values increased significantly. Conversely, in the non-improved group with baseline $\mathrm{EF} \geq 50 \%$, both non-gated \%uptake and ES \%uptake values did not increase. In patients with EF $<50 \%$, however, although \%uptake was slightly increased in the non-improved group, EF and \% wall thickening did not change significantly.

When predictors of EF improvement $>5 \%$ were analyzed, the optimal minimal \%uptake was $41 \%$ for the non-gated map and 34\% for ES \%uptake (Table 6). Based on these threshold criteria of \%uptake, sensitivity and specificity were $57 \%$ and $62 \%$ for non-gated \%uptake values, while they were $54 \%$ and $64 \%$ for ES \%uptake. The optimal number of viable segments was defined as $\geq 2$ segments of \%uptake $>63 \%$ for non-gated map, and $\geq 3$ segments of \%uptake $>52 \%$ for ES map. When number of viable segments was used as the predictor of improvement in EF, sensitivity and specificity were $67 \%$ and $48 \%$ for non-gated \%uptake and $60 \%$ and $55 \%$ for ES \%uptake. Negative predictive value was $0.72-0.73$ for both non-gated and ES \%uptake. The AUC ranged from 0.58-0.60.

\section{Discussion}

This study was a multicenter cohort study for viability assessment using quantitative gated SPECT involving 25 hospitals. We confirmed the role of ${ }^{99 \mathrm{~m}}$ Tc-MIBI gated SPECT for predicting functional 
recovery after myocardial infarction. Quantitative assessment derived from ES perfusion and non-gated perfusion maps showed higher diagnostic accuracy compared with the conventional visual assessment of perfusion defect scoring. Subanalysis with respect to the number of revascularized coronary vessels, coronary territory and revascularization type showed comparable diagnostic accuracy for viability assessment. As a predictor of functional recovery, the aid of quantitative assessment with gated SPECT showed a higher diagnostic value than visual segmental evaluation, and would be recommended particularly for multi-center studies.

Precedent studies on viability assessment

Assessment of myocardial viability after myocardial infarction has been considered vital for indicating coronary revascularization, and a number of studies have evaluated predictive values of ${ }^{99 \mathrm{~m}} \mathrm{Tc}-\mathrm{MIBI}$ for wall motion recovery in the US and European counties [8, 12, 22-24]. Histological basis was given by Maes et al., who found a linear relation between percent fibrosis and MIBI uptake [10]. Considering that Japanese patients have different backgrounds for risk factors and lower cardiac hard event rate, we designed this multi-center cohort study with a larger number of patients $[25,26]$. Our study showed that sensitivity and specificity of functional recovery by non-gated \%uptake were $68 \%$ and $64 \%$, and those of ES \%uptake were $80 \%$ and $52 \%$, showing higher sensitivity of $\% \mathrm{ES}$ uptake. According to the meta-analysis by Bax et al. sensitivity and specificity of SPECT from $7^{99 \mathrm{~m}} \mathrm{Tc}-\mathrm{MIBI}$ studies were $81 \%(191 / 236)$ and 60\% (122/203) [5]. Specificity varied from $35 \%$ to $86 \%$ in spite of a high sensitivity from $73 \%$ to $100 \%$. The present study also showed comparable values for diagnostic accuracy, and slightly different characteristics of each quantitative predictor.

\section{Characteristics of the present study}

This study was based on a multi-center cohort study involving 25 Japanese institutions and 252 patients. The predictive values of AUC, sensitivity and specificity were comparable or slightly lower than the precedent investigations $[5,8,12]$. However, ours were comparable with the results by Acampa et al. using sublingual nitrate administration [9]. Few studies have used 20-segment-based wall motion evaluation and a 5 -grade scoring method in viability studies. From the SPECT image interpretation to assessment of predictors, we used aids of displays from quantitative software, here with QGS and QPS. Since many institutions participated in this study, some differences in preferences for acquisition and processing should be overcome by the use of quantitative software [27].

The demographics of the patients showed less severe profiles compared with those of the precedent studies. Mean EFs were $34 \pm 10 \%$ by Udelson et al. [8] and $36 \pm 9 \%$ by Kang et al. [12], and those in most studies were from $30 \%$ to $50 \%$ [5]. In contrast, our study population showed mean EF of $50 \%$ and mean EDV of $103 \mathrm{~mL}$ before revascularization, average segmental \%uptake of $66 \%$ and $55 \%$ for improved and non-improved groups, and mean visual perfusion defect score of 1 and 1.3 for improved and non-improved groups, respectively. In addition, $56 \%$ of patients had one-vessel disease. The less severe profile might have resulted in a modest predictive accuracy.

Optimal threshold values

The optimal threshold values in this study were $63 \%$ of the peak activity for non-gated \%uptake and $52 \%$ for ES \%uptake. Udelson et al. found that ${ }^{201} \mathrm{Tl}$ and ${ }^{99 \mathrm{~m}}$ Tc-MIBI regional activities were similar in segments with reversible $(72 \pm 11 \%$ versus $75 \pm 9 \%$, respectively) as well as irreversible ventricular dysfunction $(51 \pm 11 \%$ versus $50 \pm 8 \%)$ [8]. The cutoff value to separate reversible from irreversible segments was approximately $60 \%$. A difference of $25 \%$ of peak activity between reversible and irreversible segments was found in their study, while our study showed a difference of $11 \%$ by non-gated polar map. Acampa et al. also found that the optimal threshold values to separate reversible from irreversible dysfunction, as determined by ROC analysis, were $55 \%$ of peak activity for both tetrofosmin and MIBI and $60 \%$ for ${ }^{201} \mathrm{Tl}$ [9]. Maes et al. also found an optimal threshold value of $50 \%$ for prediction of functional recovery [10]. We could note that the optimal threshold of ED \%uptake was lower (43\%), and the diagnostic ability was lower than that of ES \%uptake. Thus, we should have referred to the average count derived from the ES map, although the use of ES \%uptake is not so common.

\section{Subgroup analysis for predicting functional recovery}

The subgroup analyses for the number of stenotic arteries, the location of revascularized coronary arteries and type of revascularization showed similar AUC by non-gated and ES \%uptake values, indicating the validity of these predictors for viability assessment. When we observed the coronary artery territory by non-gated \%uptake perfusion map, AUC of the LCX was lower than that of the LAD and RCA regions, whereas ES \%uptake showed comparable values for all coronary regions. Therefore, use of ES \%uptake seemed to enhance the diagnostic ability for predicting wall motion recovery of the LCX region. Ibrahim et al. also reported that sensitivity of MIBI SPECT for detecting old myocardial infarction was slightly lower in the LCX territory (79\%), compared with LAD (89\%) and RCA $(87 \%)$ territories. This might partly be explained by the underestimation of LCX ischemia by the anatomically smaller perfusion area or relatively high uptake in the lateral wall in non-attenuation corrected images. Moreover, considering image resolution of conventional SPECT systems with a full-width at half-maximum of $\sim 15 \mathrm{~mm}$, partial volume effect is smaller in the ES phase than in the ED phase or non-gated images. The blurring of images due to a larger motion of the free wall may also be a concern.

Predictors of improvement in global function Few studies have evaluated the global 
functional improvement as detected by EF [5]. Improvement of global EF was clinically important as well as recovery of regional wall motion. Therefore, we investigated the predictive value of myocardial perfusion to achieve $>5 \%$ of EF improvement. The AUCs for non-gated and ES \%uptake values were $\sim 0.60$ for predicting EF improvement, whereas they were $\sim 0.70$ for predicting regional improvement. This indicated that regional improvement did not readily reflect global functional improvement.

Slight increase in myocardial perfusion might not result in significant improvement in wall motion. Table 5 showed that in patients with EF $<50 \%$, \%uptake was slightly increased, but EF and wall thickening were not. In this respect, importance of the amount of viable myocardium was also demonstrated [12]. They found that the number of viable segments in rest ${ }^{201} \mathrm{Tl}$ uptake was a predictor of improvement, which was associated with $20-25 \%$ of the viable myocardium. The number of viable segment required for functional recovery was 2-3 segments for each coronary artery territory in our study, which corresponded to $30-40 \%$ area of each coronary artery territory, and showed comparable AUC values with \%minimal uptake per segment.

The background of the patients was also concerned. Only one third of the patients showed improvement in $\mathrm{EF}>5 \%$, and $\mathrm{OMI}$ was observed in approximately $70 \%$ of the patients in our study population. Although jeopardized myocardium after ischemic episodes could recover after appropriate therapy, the completed infarction might not have benefited directly from the revascularization. The follow-up period was not long enough (132 days) to obtain final outcome of revascularization, and further improvement in global function may have been possible after 4 months. Finally, the fundamental issue depends on the definition of viability, namely myocardial perfusion restoration and thickening improvement may not have been identical [28].

\section{Limitations}

The first limitation was concerned with the multi-center study involving 25 hospitals. Although standard acquisition protocol was used in each hospital, the technical and protocol-based preferences among institutions differed to some extent. The effect of revascularization procedures regarding $\mathrm{PCI}$ and $\mathrm{CABG}$ was also averaged in many hospitals. However, this study reflected actual clinical practice of revascularization in Japan. Therefore, although the diagnostic accuracy was slightly lower than that of the precedent studies, the variability reflected one of the worst scenarios from the viewpoint of technical concordance. The superiority of the quantitative method was also explained by the difference in preference and interpretation of the diverse quality images. In addition, restenosis was confirmed by coronary arteriography in $71 \%$ of the patients, which may partly have influenced the results. However, since the number of patients in whom no restenosis was confirmed was 87 (69\%) for the improved group and $118 \quad(72 \%)$ for the non-improved group, no bias for either of the groups was observed.

We used 20-segment model for quantification that was validated well at that time, and the model depended on the software version. However, comparable results will be obtained by the 17 -segment model [15]. The non-improved group included patients with normal EF, and those with relatively high EF before revascularization might not show significant increase in EF after revascularization. In other words, revascularization procedure would be more beneficial for patients with low EF.

In spite of these limitations, we confirmed the usefulness of gated SPECT for functional recovery in the multicenter study. The quantitative evaluation was particularly helpful when many institutions were involved.

\section{Conclusion}

Gated SPECT was performed at rest using ${ }^{99 \mathrm{~m}}$ Tc-MIBI in 252 patients from 25 institutions. The predictive accuracy of myocardial uptake for regional and global left ventricular functional improvement was evaluated. A higher predictive accuracy was obtained by ES \%uptake and non-gated \%uptake than visual scoring of perfusion defects. Subgroups analysis regarding coronary artery stenosis, the number of revascularized vessels and types of revascularization showed comparable diagnostic performances. Thus, application of quantitative gated SPECT information enhanced diagnostic accuracy and is recommended particularly for multicenter studies.

\section{References}

1. Dilsizian V, Arrighi JA, Diodati JG, Quyyumi AA, Alavi K, Bacharach SL, et al. Myocardial viability in patients with chronic coronary artery disease. Comparison of ${ }^{99 \mathrm{~m}} \mathrm{Tc}$-sestamibi with thallium reinjection and [18F]fluorodeoxyglucose. Circulation 1994;89:578-87.

2. vom Dahl J, Altehoefer C, Sheehan FH, Buechin P, Schulz G, Schwarz ER, et al. Effect of myocardial viability assessed by technetium-99m-sestamibi SPECT and fluorine-18-FDG PET on clinical outcome in coronary artery disease. $J$ Nucl Med 1997;38:742-8.

3. Tamaki N, Kawamoto M, Takahashi N, Yonekura Y, Magata Y, Nohara R, et al. Prognostic value of an increase in fluorine-18 deoxyglucose uptake in patients with myocardial infarction: comparison with stress thallium imaging. J Am Coll Cardiol 1993;22:1621-7.

4. Lucignani G, Paolini G, Landoni C, Zuccari M, Paganelli G, Galli L, et al. Presurgical identification of hibernating myocardium by combined use of technetium- $99 \mathrm{~m}$ hexakis 2-methoxyisobutylisonitrile single photon emission tomography and fluorine-18 fluoro-2-deoxy-D-glucose positron emission tomography in patients with coronary artery disease. Eur J Nucl Med 1992;19:874-81.

5. Bax JJ, Wijns W, Cornel JH, Visser FC, Boersma E, Fioretti PM. Accuracy of currently available techniques for prediction of functional recovery after revascularization in patients with left ventricular dysfunction due to chronic 
coronary artery disease: comparison of pooled data. $\mathrm{J} \mathrm{Am}$ Coll Cardiol 1997;30:1451-60.

6. Arnese M, Cornel JH, Salustri A, Maat A, Elhendy A, Reijs AE, et al. Prediction of improvement of regional left ventricular function after surgical revascularization. A comparison of low-dose dobutamine echocardiography with ${ }^{201}$ Tl single-photon emission computed tomography. Circulation 1995;91:2748-52.

7. Udelson JE. Choosing a thallium-201 or technetium $99 \mathrm{~m}$ sestamibi imaging protocol. $J \mathrm{Nucl}$ Cardiol 1994;1:S99-108.

8. Udelson JE, Coleman PS, Metherall J, Pandian NG, Gomez AR, Griffith JL, et al. Predicting recovery of severe regional ventricular dysfunction. Comparison of resting scintigraphy with $201 \mathrm{Tl}$ and $99 \mathrm{mTc}$-sestamibi. Circulation 1994;89:2552-61.

9. Acampa W, Cuocolo A, Petretta M, Bruno A, Castellani M, Finzi A, et al. Tetrofosmin imaging in the detection of myocardial viability in patients with previous myocardial infarction: comparison with sestamibi and Tl-201 scintigraphy. J Nucl Cardiol 2002;9:33-40.

10. Maes AF, Borgers M, Flameng W, Nuyts JL, van de Werf F, Ausma JJ, et al. Assessment of myocardial viability in chronic coronary artery disease using technetium-99m sestamibi SPECT. Correlation with histologic and positron emission tomographic studies and functional follow-up. $J$ Am Coll Cardiol 1997;29:62-8.

11. Marzullo P, Sambuceti G, Parodi O. The role of sestamibi scintigraphy in the radioisotopic assessment of myocardial viability. J Nucl Med 1992;33:1925-30.

12.Kang WJ, Lee DS, Paeng JC, Kim KB, Chung JK, Lee MC. Prognostic value of rest ${ }^{201}$ Tl-dipyridamole stress ${ }^{99 \mathrm{~m}}$ Tc-sestamibi gated SPECT for predicting patient-based clinical outcomes after bypass surgery in patients with ischemic left ventricular dysfunction. $J \mathrm{Nucl}$ Med 2003;44:1735-40.

13. Germano G, Erel J, Lewin H, Kavanagh PB, Berman DS. Automatic quantitation of regional myocardial wall motion and thickening from gated technetium-99m sestamibi myocardial perfusion single-photon emission computed tomography. J Am Coll Cardiol 1997;30:1360-7.

14. Berman DS, Kiat H, Friedman JD, Wang FP, van Train K, Matzer L, et al. Separate acquisition rest thallium-201/stress technetium-99m sestamibi dual-isotope myocardial perfusion single-photon emission computed tomography: a clinical validation study. $J$ Am Coll Cardiol 1993;22:1455-64.

15. Berman DS, Abidov A, Kang X, Hayes SW, Friedman JD, Sciammarella MG, et al. Prognostic validation of a 17-segment score derived from a 20-segment score for myocardial perfusion SPECT interpretation. J Nucl Cardiol 2004;11:414-23.

16. Kumita S, Kumazaki T. Assessment of left ventricular function with ${ }^{99 \mathrm{~m}}$ Tc-MIBI gated myocardial SPECT using 3 head rotating gamma camera (In Japanese). Kaku Igaku (Jpn J Nucl Med) 1994;31:43-52.

17. Shirakawa S, Hattori N, Tamaki N, Fujita T, Yano S, Kudoh $\mathrm{T}$, et al. Assessment of left ventricular wall thickening with gated ${ }^{99 \mathrm{~m}}$ Tc-MIBI SPECT--value of normal file (in Japanese)). Kaku Igaku (Jpn J Nucl Med) 1995;32:643-50.

18. Giubbini R, Rossini P, Bertagna F, Bosio G, Paghera B, Pizzocaro C, et al. Value of gated SPECT in the analysis of regional wall motion of the interventricular septum after coronary artery bypass grafting. Eur J Nucl Med Mol Imaging 2004;31:1371-7.

19. Taki J, Higuchi T, Nakajima K, Matsunari I, Hwang EH, Bunko $\mathrm{H}$, et al. Electrocardiographic gated ${ }^{99 \mathrm{~m}} \mathrm{Tc}-\mathrm{MIBI}$
SPECT for functional assessment of patients after coronary artery bypass surgery: comparison of wall thickening and wall motion analysis. $J$ Nucl Med 2002;43:589-95.

20.Fleiss J, Cohen J. The equivalence of weighted kappa and the intraclass correlation coefficient as measures of reliability. Educ Psychol Meas 1973;33:613-9.

21.DeLong ER, DeLong DM, Clarke-Pearson DL. Comparing the areas under two or more correlated receiver operating characteristic curves: a nonparametric approach. Biometrics 1988;44:837-45.

22.Bax JJ, Maddahi J, Poldermans D, Elhendy A, Cornel JH, Boersma E, et al. Sequential ${ }^{201} \mathrm{Tl}$ imaging and dobutamine echocardiography to enhance accuracy of predicting improved left ventricular ejection fraction after revascularization. $J$ Nucl Med 2002;43:795-802.

23. Marzullo P, Sambuceti G, Parodi O, Gimelli A, Picano E, Giorgetti A, et al. Regional concordance and discordance between rest thallium 201 and sestamibi imaging for assessing tissue viability: comparison with postrevascularization functional recovery. $\mathrm{J} \mathrm{Nucl} \mathrm{Cardiol}$ 1995;2:309-16.

24.Bisi G, Sciagra R, Santoro GM, Rossi V, Fazzini PF. Technetium-99m-sestamibi imaging with nitrate infusion to detect viable hibernating myocardium and predict postrevascularization recovery. $J \quad \mathrm{Nucl}$ Med 1995;36:1994-2000.

25.Kawano H, Soejima H, Kojima S, Kitagawa A, Ogawa H. Sex differences of risk factors for acute myocardial infarction in Japanese patients. Circ J 2006;70:513-7.

26. Saito M, Fukami K, Hiramori K, Haze K, Sumiyoshi T, Kasagi H, et al. Long-term prognosis of patients with acute myocardial infarction: is mortality and morbidity as low as the incidence of ischemic heart disease in Japan. Am Heart $J$ 1987;113:891-7.

27. Nakajima K, Nishimura T. Inter-institution preference-based variability of ejection fraction and volumes using quantitative gated SPECT with 99mTc-tetrofosmin: a multicentre study involving 106 hospitals. Eur J Nucl Med Mol Imaging 2006;33:127-33.

28. Kaul S. There may be more to myocardial viability than meets the eye! Circulation 1995;92:2790-3.

\section{Appendix}

List of the hospitals for MIBI myocardial viability research group follows.

Sapporo Medical University Hospital, Hokkaido University Hospital, Wakkanai City Hospital, Hokko Memorial Hospital, Kushiro City Medical Association Hospital, Yamagata University Hospital, Hoshi General Hospital, Toho University Omori Hospital Center, Toranomon Hospital, Sakakibara Commemoration Hospital, Shonan Kamakura General Hospital, Tomioka General Hospital, Nagoya Daini Red Cross Hospital, Social Insurance Hamamatsu Hospital, Kansai Medical University Takii Hospital, National Cardiovascular Center, Kyoto University Hospital, Kyoto University Hospital, Kanazawa University Hospital, Koseiren Takaoka Hospital, Fukui Prefectural Hospital, Hokuriku Central Hospital of Japan Mutual Aid Association of Public School Teachers, Kanazawa Cardiovascular Hospital, Saiseikai Saijo Hospital, National Hospital Organization Ehime National Hospital, Saiseikai Kumamoto Hospital 


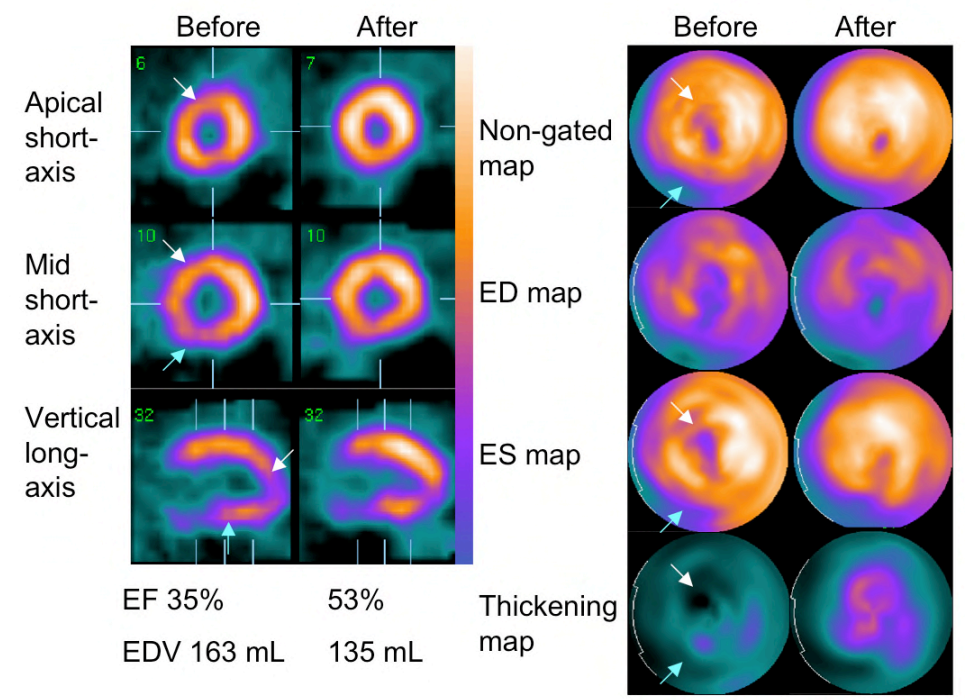

Fig. 1

A 70-year-old male patient with old myocardial infarction in the anteroseptal and inferior walls. RCA mid segment (segment 2) showed stenosis of $90 \%$ and was improved to $0 \%$ after stenting. Proximal LAD stenosis (segment 6 ) of $75 \%$ was also improved to $0 \%$ after stenting. Stenosis of LCX posterolateral branch (segment 14, 75\%) was not revascularized. Before revascularization, mid to apical anteroseptal regions (white arrows) and the inferior region (blue arrows) showed hypoperfusion, which corresponded to the LAD and RCA stenosis. Changes in perfusion and thickening were evident in the ES perfusion and wall thickening maps, particularly in the anteroseptal region. Improvement in global EF and volumes could be also noted.
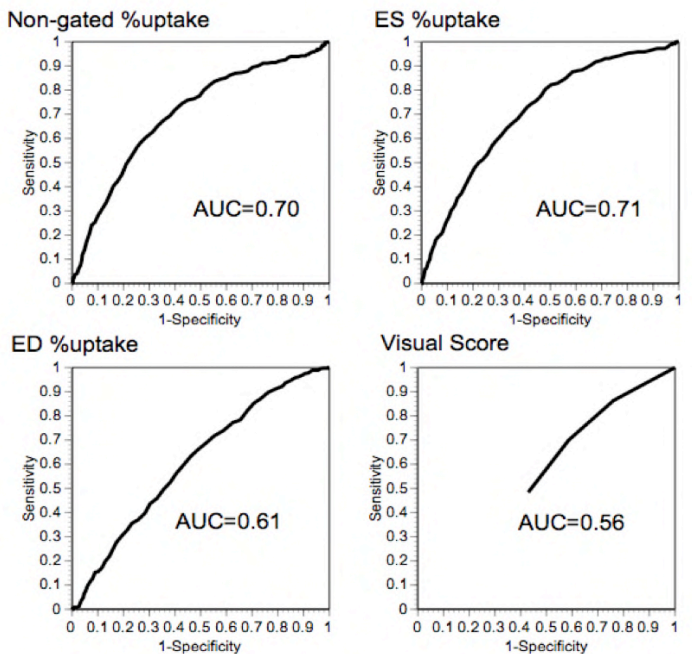

Fig. 2

ROC curves for \%uptake on non-gated perfusion, end-systolic (ES) perfusion and end-diastolic (ED) perfusion maps, and visual perfusion score for predicting functional improvement of segmental asynergy after revascularization. The AUCs were 0.70 (95\% CI, 0.67-0.73), $0.71(0.68-0.74), 0.61(0.58-0.64)$ and 0.56 (0.52-0.59), respectively. 
Table 1 Demographics of 252 patients

\begin{tabular}{llll}
\hline & & number & $\%$ \\
\hline Gender & male & 216 & $86 \%$ \\
Type of infarction & RMI & 74 & $29 \%$ \\
No. of diseased vessels & OMI & 175 & $69 \%$ \\
& RMI and OMI & 3 & $1 \%$ \\
Type of revascularization & 1 & 140 & $56 \%$ \\
& 2 & 59 & $23 \%$ \\
Stenotic lesion & 3 & 53 & $21 \%$ \\
& PCI & 210 & $83 \%$ \\
& CABG & 40 & $16 \%$ \\
& PCI and CABG & 2 & $1 \%$ \\
& LAD & 150 & $60 \%$ \\
& LCX & 70 & $28 \%$ \\
\hline
\end{tabular}

Abbreviations: RMI, recent myocardial infarction; OMI , old myocardial infarction; PCI, percutaneous coronary intervention; CABG, coronary artery bypass grafting; $\mathrm{LAD}$, left anterior descending coronary artery; LCX, left circumflex coronary artery; RCA, right coronary artery

Table 2 Comparison of viability predictors between segment groups with and without regional improvement before and after revascularization

\begin{tabular}{llll}
\hline Predictors & Improved & Not improved & P value (unpaired) \\
\hline $\begin{array}{l}\text { Number of segments } \\
\text { ouptake on non-gated map }\end{array}$ & 360 & 1370 & \\
Before & $66 \pm 17$ & $55 \pm 18$ & $<0.0001$ \\
After & $75 \pm 11$ & $58 \pm 17$ & $<0.0001$ \\
P value (paired) & $<0.0001$ & $<0.0001$ & \\
\%uptake on ES map & & & $<0.0001$ \\
Before & $64 \pm 16$ & $51 \pm 17$ & $<0.0001$ \\
After & $74 \pm 11$ & $54 \pm 16$ & \\
P value (paired) & $<0.0001$ & $<0.0001$ & $<0.0001$ \\
$\%$ ouptake on ED map & & & $<0.0001$ \\
Before & $47 \pm 12$ & $42 \pm 13$ & \\
After & $50 \pm 9$ & $45 \pm 13$ & $<0.0001$ \\
P value (paired) & $<0.0001$ & $<0.0001$ & \\
$\%$ wall-thickening on polar & map & & \\
Before & $30 \pm 13$ & $16 \pm 10$ & \\
After & $44 \pm 12$ & $17 \pm 9$ & \\
P value (paired) & $<0.0001$ & $<0.0001$ & \\
\hline Abbreviations & end-systocis & &
\end{tabular}

Abbreviations: ES, end-systocic; ED, end-diastoic 
Table 3 ROC analyses and AUC in subgroups of patients

\begin{tabular}{cllllll}
\hline & \multicolumn{2}{c}{ No. of segments } & \multicolumn{2}{c}{ non-gated \% uptake } & \multicolumn{2}{c}{ ES \% uptake } \\
& $(\mathrm{n}=1730)$ & AUC & $95 \% \mathrm{CI}$ & AUC & $95 \% \mathrm{CI}$ \\
\hline Types of infarction & & & & & \\
RMI & 482 & 0.66 & $0.59-0.73$ & 0.69 & $0.63-0.75$ \\
OMI & 1248 & 0.71 & $0.68-0.74$ & 0.72 & $0.68-0.75$ \\
\multicolumn{2}{rl}{ Revascularized artery } & & & & \\
LAD & 960 & 0.71 & $0.67-0.75$ & 0.71 & $0.68-0.75$ \\
LCX & 296 & 0.66 & $0.57-0.75$ & 0.70 & $0.62-0.78$ \\
RCA & 474 & 0.73 & $0.68-0.80$ & 0.73 & $0.67-0.79$
\end{tabular}

No. of revascularized artery

$\begin{array}{llllll}1 \text { vessel } & 735 & 0.72 & 0.68-0.76 & 0.74 & 0.71-0.78 \\ 2 \text { vessels } & 404 & 0.71 & 0.65-0.77 & 0.72 & 0.66-0.78 \\ 3 \text { vessels } & 591 & 0.71 & 0.65-0.77 & 0.70 & 0.64-0.76\end{array}$

Type of revascularization

$\begin{array}{llllll}\text { PCI } & 1223 & 0.70 & 0.64-0.77 & 0.73 & 0.70-0.76 \\ \text { CABG } & 507 & 0.71 & 0.68-0.75 & 0.70 & 0.63-0.76\end{array}$

Abbreviations: ROC, receiver operating characteristics; AUC, area under the curve; $\mathrm{CI}$, confidence interval

Table 4 Diagnostic accuracy and AUC of \% uptake for predicting regional functional improvement after revascularization

\begin{tabular}{lll}
\hline Patient-based statistics & Non-gated map & ES map \\
\hline Optimal threshold for \%uptake* & $63 \%$ & $52 \%$ \\
Sensitivity & $68 \%(243 / 360)$ & $80 \%(289 / 360)$ \\
Specificity & $64 \%(882 / 1370)$ & $52 \%(712 / 1370)$ \\
PPV & $33 \%(243 / 731)$ & $31 \%(289 / 947)$ \\
NPV & $88 \%(882 / 999)$ & $91 \%(712 / 783)$ \\
Accuracy & $65 \%(1125 / 1730)$ & $58 \%(1001 / 1730)$ \\
AUC & 0.70 & 0.71 \\
\hline
\end{tabular}

* the segmental \%uptake to the maximal count

Abbreviations: PPV, positive predictive value; NPV, negative predictive value; AUC, area under the curve 
Table 5 Comparison of viability predictors and LVEF between patient groups with and without global EF improvement (>5\% EF unit)

\begin{tabular}{|c|c|c|c|c|}
\hline & \multicolumn{2}{|c|}{ Baseline EF $\geq 50 \%$} & \multicolumn{2}{|c|}{ Baseline $\mathrm{EF}<50 \%$} \\
\hline & Improved & Not improved & Improved & Not improved \\
\hline Number of patients & $39(28 \%)$ & $100(72 \%)$ & $48(42 \%)$ & $65(58 \%)$ \\
\hline \multicolumn{5}{|c|}{ \%uptake of the minimal segment on non-gated map } \\
\hline Before & $42 \pm 14$ & $48 \pm 12$ & $35 \pm 18$ & $35 \pm 15$ \\
\hline After & $48 \pm 12$ & $49 \pm 11$ & $42 \pm 15$ & $40 \pm 16$ \\
\hline$P$ value (paired) & 0.0068 & n. s. & 0.0016 & 0.0004 \\
\hline \multicolumn{5}{|c|}{ \%uptake of the minimal segment on ES map } \\
\hline Before & $37 \pm 9$ & $42 \pm 11$ & $31 \pm 15$ & $33 \pm 12$ \\
\hline After & $42 \pm 12$ & $43 \pm 10$ & $39 \pm 13$ & $36 \pm 13$ \\
\hline$P$ value (paired) & 0.0014 & n. s. & 0.0006 & 0.0037 \\
\hline \multicolumn{5}{|l|}{$E F(\%)$} \\
\hline Before & $58 \pm 7$ & $62 \pm 8$ & $35 \pm 10$ & $39 \pm 9$ \\
\hline After & $68 \pm 7$ & $60 \pm 9$ & $47 \pm 12$ & $38 \pm 9$ \\
\hline$P$ value (paired) & $<0.0001$ & $<0.0001$ & $<0.0001$ & n. s. \\
\hline \multicolumn{5}{|c|}{$\%$ wall thickening of the minimal segment } \\
\hline Before & $12 \pm 8$ & $16 \pm 9$ & $3 \pm 6$ & $4 \pm 6$ \\
\hline After & $20 \pm 13$ & $14 \pm 8$ & $10 \pm 7$ & $4 \pm 6$ \\
\hline P value (paired) & $<0.0001$ & 0.0079 & $<0.0001$ & n. s. \\
\hline
\end{tabular}

Abbreviation: EF, ejection fraction

Table 6 Diagnostic accuracy and AUC of \%uptake and number of viable segments for predicting EF improvement $>5 \%$ after revascularization

\begin{tabular}{lllll}
\hline \multirow{2}{*}{$\begin{array}{l}\text { Patient-based } \\
\text { statistics }\end{array}$} & \multicolumn{2}{l}{ The minimal \% uptake/segment } & \multicolumn{2}{l}{ No. of viable segments } \\
\hline Optimal threshold & $41 \% *$ & Non-gated map & ES map \\
Sensitivity & $57 \%(50 / 87)$ & $54 \%(47 / 87)$ & $67 \%(52 / 87)$ & $\geq 3$ segments*** \\
Specificity & $62 \%(102 / 165)$ & $64 \%(106 / 165)$ & $48 \%(91 / 165)$ & $60 \%(52 / 87)$ \\
PPV & $44 \%(50 / 113)$ & $44 \%(47 / 106)$ & $41 \%(58 / 143)$ & $41 \%(52 / 126)$ \\
NPV & $73 \%(102 / 139)$ & $73 \%(106 / 146)$ & $73 \%(80 / 109)$ & $72 \%(91 / 126)$ \\
Accuracy & $60 \%(152 / 252)$ & $61 \%(153 / 252)$ & $55 \%(138 / 252)$ & $57 \%(143 / 252)$ \\
AUC & 0.60 & 0.60 & 0.59 & 0.58 \\
\hline$*$ the minimal segmental \%uptake to the maximal count, ** the viable segment with non-gated $\%$ uptake of $>63 \%, * * *$
\end{tabular}

